\title{
Predictive modeling of surface and dimensional features of vapour-smoothened FDM parts using self-adaptive cuckoo search algorithm
}

\author{
Jasgurpreet Singh Chohan ${ }^{1}$ (1) Nitin Mittal ${ }^{2} \cdot$ Rupinder Singh $^{3} \cdot$ Urvinder Singh $^{4} \cdot$ Rohit Salgotra $^{5} \cdot$ Raman Kumar $^{1}$. \\ Sandeep Singh ${ }^{6}$
}

Received: 16 July 2021 / Accepted: 13 February 2022 / Published online: 6 March 2022

(c) The Author(s), under exclusive licence to Springer Nature Switzerland AG 2022

\begin{abstract}
Despite numerous advantages of fused deposition modeling (FDM), the inherent layer-by-layer deposition behavior leads to considerable surface roughness and dimensional variability, limiting its usability for critical applications. This study has been conducted to select optimum parameters of FDM and vapour smoothing (chemical finishing) process to maximize surface finish, hardness, and dimensional accuracy. A self-adaptive cuckoo search algorithm for predictive modelling of surface and dimensional features of vapour-smoothened FDM-printed functional prototypes has been demonstrated. The chemical finishing has been performed on hip prosthesis (benchmark) using hot vapours of acetone (using dedicated experimental set-up). Based upon the selected design of experiment technique, 18 sets of experiments (with three repetitions) were performed by varying six parameters. Afterwards, a self-adaptive cuckoo search algorithm was implemented by formulating five objective functions using regression analysis to select optimum parameters. An excellent functional relationship between output and input parameters has been developed using a self-adaptive cuckoo search algorithm which has successfully found the solution to optimization issues related to different responses. The confirmatory experiments indicated a strong correlation between predicted and actual surface finish measurements, along with hardness and dimensional accuracy.
\end{abstract}

Keywords Fused deposition modeling $\cdot$ Self-adaptive cuckoo search algorithm $\cdot$ Surface finish $\cdot$ Hardness $\cdot$ Dimensional accuracy

Jasgurpreet Singh Chohan

jaskhera@gmail.com

1 Department of Mechanical Engineering, Chandigarh University, Mohali 140413, India

2 Department of Electronics and Communication Engineering, Chandigarh University, Mohali 140413, India

3 Department of Mechanical Engineering, National Institute of Technical Teachers Training and Research, Chandigarh 160019, India

4 Department of Electronics and Communication Engineering, Thapar Institute of Engineering and Technology, Patiala 147004, India

5 Tel Aviv University, Tel Aviv, Israel

6 Department of Civil Engineering, Chandigarh University, Mohali 140413, India

\section{Introduction}

FDM is currently utilized to fabricate functional prototypes, visual aids, and conceptual designs and used for the fabrication of biomedical implants, auto-parts, and aircraft engine components. The unique significance of this technology is its capability to manufacture complex designs within minimum time and cost [1]. The added feature of this technology is the wide range and flexibility of materials which are thermoplastics and their composites [2]. Despite these advantages, researchers and industries do not universally adopt the technology due to certain process limitations, such as poor surface finish, dimensional accuracy, and mechanical strength [3]. In FDM process, the thermoplastic print material is heated slightly lower than its melting point and passed through the "Print head" which is numerically controlled. Rollers inside "Print head" control the movement of thermoplastic print material; finally, semi-molten print material is extruded by a nozzle on the build tray [4]. 
Despite wide use for Rapid Prototyping, FDM process has been used for various applications, such as aerospace, electronics, product development, pharmaceutical and biomedical [5]. The role of this technology has been further highlighted during COVID-19 outbreak where rapid designing and development of medical equipment were realized [6]. The production of face shields, facemasks, splitter valves, venturi valves, mask pleaters and many more devices has been intensified using Additive manufacturing technologies during the pandemic [7]. Recent studies have reported the use organic waste utilization through FDM technology for biomedical industry. Naturally available Polylactic acid was mixed with $20 \%$ agriculture waste (Hemp and Weed) to prepare feedstock filament for FDM which was further used to fabricate neck orthosis [8]. For electronics industry, the metamaterials-based sensors and EMI shielding devices were prepared using FDM technology. The solvent sensing capability of printed conductive polymer was successfully demonstrated [9]. However, this technology has some inherent limitations, such as surface finish and dimensional accuracy, which may need post-processing of parts. The surface roughness of FDM parts is a significant issue faced by researchers due to layer-by-layer deposition strategy, which is a basic principle of this technology. During stacking of different layers, the stair steps are visible, especially in curved and circular parts. In linear dimensions also, peaks and valleys (Fig. 1) are visible, which occur due to the gap between two deposited layers of print material [10].

Many scientists have implemented optimization studies where the best combination of input parameters is identified, but there is a limit beyond which surface finish cannot be enhanced due to the innate characteristics of FDM technology. Conclusively, post-processing techniques are used by researchers where products undergo various types of finishing operations [11]. Mechanical finishing ball end magnetorheological process has been implemented by Kumar et al. [12] which significantly reduced surface roughness of FDM parts. Abrasive flow machining [13] has also been used for surface enhancement of FDM parts. The process is effective but temperature rise at surface has been noted which may harm the surface integrity. An alternate method has been recently adopted by researchers where chemicals and solvents are used for the finishing of FDM parts. Jayanth et al. [14] reported an increase in tensile of DFM parts along with surface improvement using acetone. The surface finish netter enhanced by di-chloro-ethane but tensile strength of parts is compromised. Surface coating of FDM parts has also been reported by Ivan et al. [15] where pre-treated parts are blasted by sodium carbonate and glass beads. The process can be recommended for industrial grade products but cost of manufacturing may be significantly increases if surface fish is only objective. $\mathrm{CO}_{2}$ Laser polishing of PLA parts has been studied by Dewey et al. [16] and reported $97 \%$ improvement in surface quality. The impact of variable power and speed has also been studied. Roach et al. [17] tried to modify the surface of FDM-based polyetherimide parts which were intended to be used for making RF devices using conductive ink. This study demonstrated the use of poly(ethylene glycol) diacrylate below the dielectric polyimide ink for retaining the conductivity along with surface improvement.

Since various conventional and non-conventional techniques have been implemented, but the dedicated vapour finishing apparatus has been used in limited studies. The use

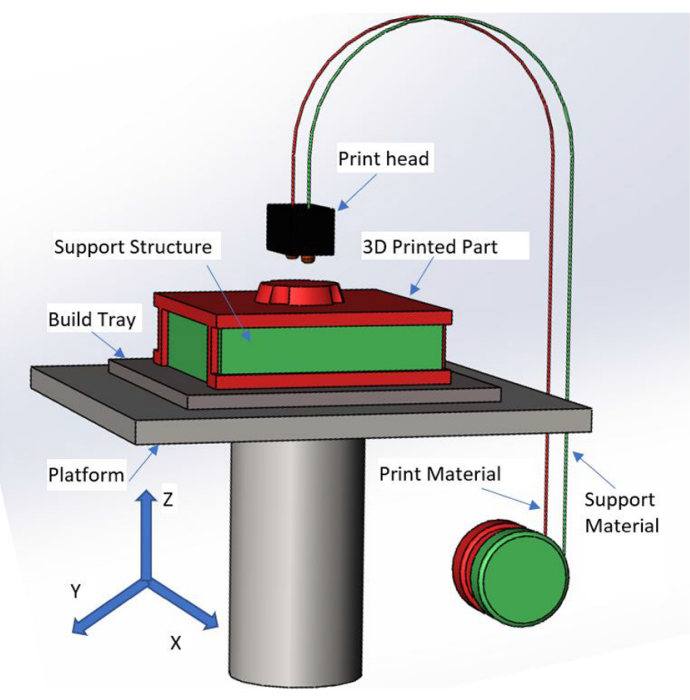

(a)

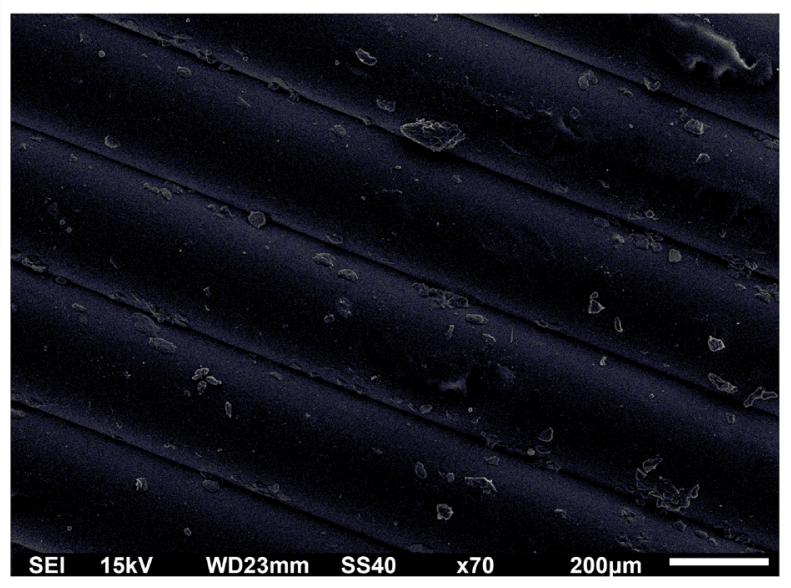

(b)

Fig. 1 a Schematic of FDM, b surface roughness visible after fabrication 
of commercial vapour finishing apparatus would give consistent results which are necessary for maintaining product quality and dimensional tolerances. Furthermore, there is need to attain optimized parametric settings of this apparatus for recommending the process for commercial production.

Previous researchers have used advanced optimization algorithms for the investigation of process parameters and reported best solutions for FDM problems [18-20]. Vahabli and Rahmati [21] performed a case study to highlight the efficiency of neural networks for medical applications using a novel combination of particle swarm and imperialist competitive algorithm. Kumar et al. [12] implemented optimization tools on combined process parameters of FDM and finishing operations. Hybrid particle swarm and bacterial foraging methods were adopted by Raju et al. [22] to optimize layer thickness, support material, orientation, and filling strategy to achieve the best mechanical properties.

Numerous optimization algorithms have utilized but complex manufacturing problems require a robust optimization tool for attaining optimum solutions. Self-adaptive cuckoo (SACS) search algorithm uses Gaussian sampling mechanism known as bare-bones variant to enhance exploration and exploitation tendencies. This technique has yielded promising results as compared to conventional algorithms and thus, there is need to implement this technique for complex manufacturing problems.

This study investigates the optimization of combined process parameters of FDM and chemical finishing process (using dedicated vapour finishing process) which would yield best results in terms of surface finish, dimensional accuracy and hardness of FDM parts. An advanced selfadaptive cuckoo search algorithm has been used to acquire optimum solutions.

\section{Experimentation}

The replica of hip prosthesis was prepared with ABS (Grade: P400) material for analysis. The FDM printer (Model: Uprint SE) and chemical finishing apparatus (Model: Finishing Touch Smoothing Station) was used for experimentation. The parts were prepared at different settings of orientation angle (A) and infill density (B) for analysis. The chemical finishing apparatus converts liquid acetone into vapours which reacts with target surface. These vapours are again condensed as they rise upwards to avoid wastage and reused for finishing of further parts. The replica of hip implant undergoing chemical finishing process has been displayed in Fig. 2a. Initially the benchmark was hung in cooling chamber which prepares it for further processing for specific pre-cooling time (C). Afterwards, the part is hanged in smoothing chamber for exposure to chemical vapours (Fig. 2b) for pre-defined smoothing time (D). Again,

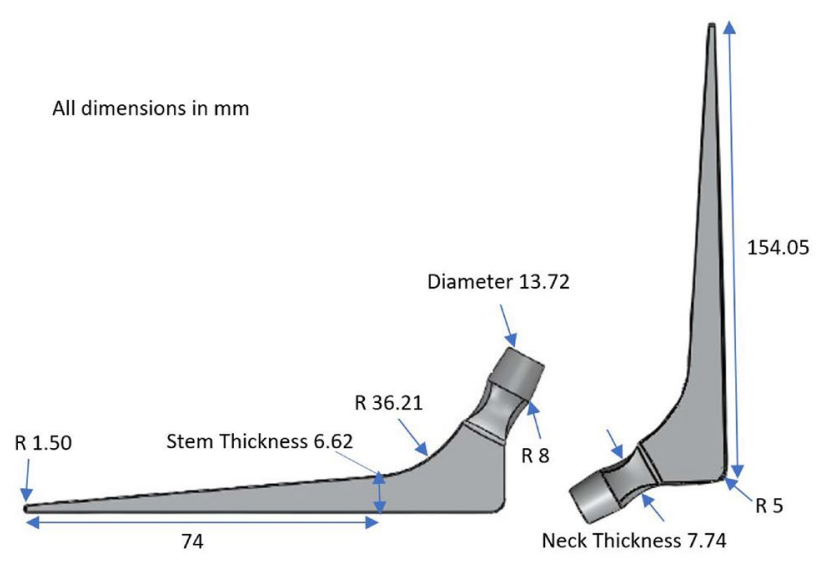

(a)

(b)

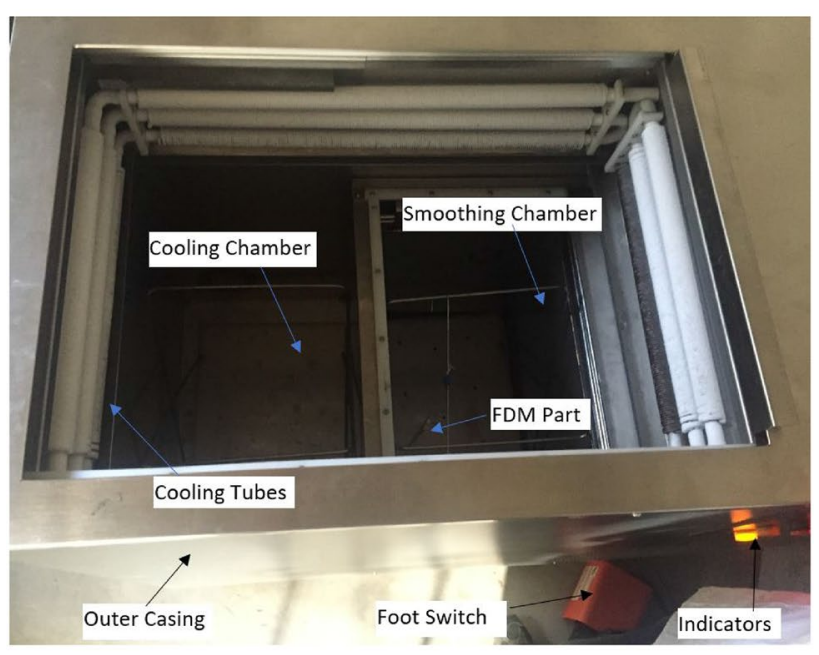

(c)

Fig. 2 a Replica at $0^{\circ}$ orientation, b replica at $90^{\circ}$ orientation, c chemical vapour finishing set-up

the specimen was placed in cooling chamber again after one finishing cycle is completed for specific post-cooling time (E) which is another parameter under study. These three steps (finishing cycles) were repeated till required finish is achieved. Hence, the last parameter is finishing cycles (F) which is a variable parameter in this study. The selection of input process parameters of FDM and chemical finishing processes are based on previous studies (Table 1).

The response parameters for present study are percentage change in surface roughness, surface hardness, diameter, neck thickness and stem thickness. The last three response parameters signify the dimensional accuracy of three important components of hip replica as shown in Fig. 2a. The surface roughness and hardness were measured at pre-specified marked location at stem of hip implant replica. Surface roughness was measured in the direction perpendicular to deposited layers. On the other hand, hardness was measured 
Table 1 Variable process parameters and their ranges used for experimentation

\begin{tabular}{ll}
\hline Parameter & Range \\
\hline A & $0-90^{\circ}$ \\
B & $0-614-0.945 \mathrm{~g} / \mathrm{cm}^{3}$ \\
C & $0-2400 \mathrm{~s}$ \\
D & $0-120 \mathrm{~s}$ \\
E & $0-2400 \mathrm{~s}$ \\
F & $1-5$ \\
\hline
\end{tabular}

using durometer on same location on stem to avoid random error. Three measurements were taken before and after finishing and average was considered as final value. The surface roughness was measured using Mitutoyo tester (Model: SJ 210) as per ISO 4287 regulations [23]. Surface hardness is measured using commercial Durometer (Model: TX 100) as per ASTM D2240 [24] whereas dimensions were measured using coordinate measuring machine (Make: CrystaApex, Model: C163012) as per ISO 10360-2 [25]. The coordinate measuring machine with $0.1 \mathrm{~mm}$ resolution tested the features of hip implant replica at 113 touch points to estimate the specified dimensions. The initial and final values of these five response parameters were used to calculate percentage change. It is desirable to maximize the change in surface roughness and hardness but minimize the changes in dimensional variables. Table 2 shows the experimental $\log$ and observations along with objective functions, i.e. maximization of percentage changes in surface roughness (R1) and hardness (R2), whereas minimization in percentage changes in dimensions of diameter (R3), neck thickness (R4) and stem thickness (R5). These data have been used as input for SACS algorithm to find an optimum solution.

\section{Self-adaptive cuckoo search algorithm (SACS)}

Nowadays, many scientific fields and industrial companies face a lot of hurdles in finding an appropriate optimization tool or more precisely an optimization algorithm to solve many real-world problems. The major reason for the use of these algorithms is that majority of the real-world applications can be formulated as potential domain optimization problems depending upon their nature and number of objectives to be optimized. This can be estimated from the fact that these algorithms find application in almost every domain of research, such as economics, engineering, mathematics, signal processing, weather forecasting, operation research, management, production planning, scheduling, routing problems, machine learning and others. These optimization problems are highly complex and hence pose a challenge for researchers to solve them in an efficient manner. In recent times, nature inspired algorithms have demonstrated better results in the area of manufacturing. Additive manufacturing is a yet new field where digital manufacturing technologies are utilized for product development.
Table 2 Experimental $\log$ and observation of surface roughness, hardness and various dimensions

\begin{tabular}{|c|c|c|c|c|c|c|c|c|c|c|c|}
\hline \multicolumn{7}{|c|}{ Design of experimentation } & \multicolumn{2}{|c|}{$\begin{array}{l}\text { Objective: maxi- } \\
\text { mize }\end{array}$} & \multicolumn{3}{|c|}{ Objective: minimize } \\
\hline Exp No & A & $\mathrm{B}$ & $\mathrm{C}$ & $\mathrm{D}$ & $\mathrm{E}$ & $\mathrm{F}$ & $\mathrm{R} 1$ & $\mathrm{R} 2$ & $\mathrm{R} 3$ & $\mathrm{R} 4$ & R5 \\
\hline 1 & 0 & 0.614 & 600 & 10 & 600 & 1 & 70.14 & 6.29 & 0.45 & 1.10 & 3.34 \\
\hline 2 & 0 & 0.614 & 900 & 15 & 900 & 2 & 82.76 & 7.63 & 0.76 & 0.63 & 1.16 \\
\hline 3 & 0 & 0.614 & 1200 & 20 & 1200 & 3 & 92.98 & 9.22 & 1.28 & 0.21 & 0.19 \\
\hline 4 & 0 & 0.820 & 600 & 10 & 900 & 2 & 75.08 & 7.84 & 0.62 & 0.94 & 2.58 \\
\hline 5 & 0 & 0.820 & 900 & 15 & 1200 & 3 & 86.87 & 8.44 & 1.30 & 0.52 & 0.62 \\
\hline 6 & 0 & 0.820 & 1200 & 20 & 600 & 1 & 87.05 & 6.45 & 1.54 & 0.26 & 0.95 \\
\hline 7 & 0 & 0.945 & 600 & 15 & 600 & 3 & 87.69 & 7.36 & 0.85 & 0.52 & 0.63 \\
\hline 8 & 0 & 0.945 & 900 & 20 & 900 & 1 & 86.46 & 7.32 & 1.39 & 0.25 & 1.62 \\
\hline 9 & 0 & 0.945 & 1200 & 10 & 1200 & 2 & 76.38 & 8.43 & 0.38 & 0.88 & 2.58 \\
\hline 10 & 90 & 0.614 & 600 & 20 & 1200 & 2 & 89.33 & 9.67 & 0.94 & 0.12 & 0.05 \\
\hline 11 & 90 & 0.614 & 900 & 10 & 600 & 3 & 82.70 & 7.53 & 0.21 & 0.19 & 0.31 \\
\hline 12 & 90 & 0.614 & 1200 & 15 & 900 & 1 & 74.67 & 8.60 & 0.25 & 0.21 & 0.24 \\
\hline 13 & 90 & 0.820 & 600 & 15 & 1200 & 1 & 75.81 & 10.57 & 0.28 & 0.15 & 0.27 \\
\hline 14 & 90 & 0.820 & 900 & 20 & 600 & 2 & 90.96 & 8.57 & 0.75 & 0.11 & 0.17 \\
\hline 15 & 90 & 0.820 & 1200 & 10 & 900 & 3 & 88.94 & 9.52 & 0.38 & 0.18 & 0.88 \\
\hline 16 & 90 & 0.945 & 600 & 20 & 900 & 3 & 93.65 & 7.69 & 0.82 & 0.05 & 0.03 \\
\hline 17 & 90 & 0.945 & 900 & 10 & 1200 & 1 & 71.08 & 10.25 & 0.26 & 0.22 & 1.12 \\
\hline 18 & 90 & 0.945 & 1200 & 15 & 600 & 2 & 83.53 & 9.09 & 0.36 & 0.17 & 0.77 \\
\hline
\end{tabular}


During processing, there are numerous parameters which may influence the product quality. This problem must be rapidly solved to reduce commercial product development time. Thus, in present study, the complex optimization problem with six FDM process variables is solved using SACS. As the study is conducted for biomedical implant fabrication, the use of highly efficient and reliable algorithm is obligatory.

A SACS algorithm based upon Weibull distribution has been followed in this study [26-28]. The first step in the SACS algorithm is initialization using a random population of $N$ cuckoos and is found using Eq. (1).

$N_{i, j}=N_{\min , j}+\operatorname{rand}(0,1) \cdot\left(N_{\min , j}-N_{\max , j}\right)$

where $i \in\{1, \ldots . N\}, j \in\{1, \ldots . . D\}$, is the $i$ th solution in the $j$ th dimension; $\operatorname{rand}(0,1)$ is uniformly distributed random number between $[0,1] ; N_{\min , j}, N_{\max , j}$ are lower bound and upper bound of the problem under consideration respectively and $D$ is the dimension size.

In this study, dual division of iterations was followed, the global search phase for first half of the iterations is given.

$x_{1}=x_{i}-A_{1}\left(C_{1} \cdot x_{\text {best }}-x_{i}^{t}\right) ; x_{2}=x_{i}-A_{2}\left(C_{2} \cdot x_{\text {best }}-x_{i}^{t}\right) ;$

$x_{3}=x_{i}-A_{3}\left(C_{3} \cdot x_{\text {best }}-x_{i}^{t}\right)$

$x_{\text {new }}^{t+1}=\frac{x_{1}+x_{2}+x_{3}}{3}$

where $x_{\text {new }}$ is the new solution and $A_{1}, A_{2}, A_{3}$ and $C_{1}, C_{2}, C_{3}$ belongs to $A$ and $C$ respectively. $A$ and $C$ are given by

$A=2 a \cdot r_{1}-a ; C=2 \cdot r_{2}$

where $a$ is a linearly decreasing in the range of $[0,2]$ and it changes with respect to iterations, $r_{1}$ and $r_{2}$ are uniformly distributed between 0 and 1 .

Here the above Eq. (3) has been derived from the grey wolf optimizer (GWO) algorithm [29] and instead of using the positions of random solutions, the best solution is used to find three near optimal neighbors. This kind of equation modification is followed to add diversity among the search agents and hence improves the exploration properties of the algorithm.

In this case, the local search phase is same as that in the basic CS algorithm [30]. The only modification is the addition of a new scaling factor $(F)$ instead of $\epsilon$ parameter of $C S$ and the new parametric equation is given by

$F_{i}^{t+1}=\frac{1}{2} \times\left(\sin (2 \pi \times\right.$ freq $\left.\times t+\pi) \times \frac{t_{\max }-t}{t_{\max }}+1\right)$ if $r_{1}>0.5$
$F_{i}^{t+1}=\frac{1}{2} \times\left(\sin (2 \pi \times\right.$ freq $\left.\times t+\pi) \times \frac{t_{\max }-t}{t_{\max }}+1\right)$ if $r_{1}<0.5$

Here freq is the sinusoidal frequency and is a fixed function, $t$ and $t_{\max }$ is the current iteration and maximum number of iterations respectively. All of these steps are to be performed for the first half of the iterations.

For second half of iterations, local and global search mechanism is followed using a differential learning mechanism. This mechanism is referred to as bare-bones mechanism and is meant for generating high quality solutions. The mechanism is based on two generalized phenomena namely cooperative search and Gaussian mutation. The general equations for both global and local search are given by the improved global search:

$g x_{i, d}=r w \times x_{\text {new }}^{t+1}+(i-r w) \times b x_{i, d}$

$l x_{i, d}=r w \times x_{\text {new }}^{t+1}+(i-r w) \times b x_{i, d}$

Another important integration of this cooperative search is the Gaussian mutation strategy. The general equation for this kind of adaptation is given by

mut $x_{t, d}=x_{d} \times(1-N(0,1)) i=1,2, \ldots, m$

Note that the dual division of population is followed for global search phase. Here for first half of the iterations, Eqs. (2) and (3) are used whereas for second half of the iterations, Eqs. (3) and (7) are used.

The third parameter which is a really important aspect of algorithm is the switching probability. This parameter helps the algorithm in shifting from global search to local search and vice versa. In present work, Weibull distributed probability adaptation was followed. The distribution consists of three basic parameters, namely shape parameter $(\beta)$, scale parameter $(\eta)$ and location parameter $(\gamma)$ given by

$f(t)=\frac{\beta}{\eta}\left(\frac{t-\gamma}{\eta}\right) \beta-1 e^{-\left(\frac{t-\gamma}{n}\right) \beta}$

For present case, the value of shape parameter is taken as 2 whereas the third parameter that is scale parameter is adapted as per the total number of iterations.

In present case, a shrinking population size reduction strategy was followed to reduce the total number of function evaluations. The shrinking population adaptation helps the algorithm in reducing population exponentially during the initial stages, static changes in the middle and finally reducing exponentially again towards the end. The general equation for this kind of adaptation is given by 
$N_{t+1}= \begin{cases}\left(1-\Delta f_{t}^{\text {best }}\right) N_{t}, & \text { if } \Delta f_{t}^{\text {best }} \leq f_{\text {max }}^{\text {best }} \\ \left(1-\Delta f_{t}^{\text {best }}\right) N_{t}, & \text { if } \Delta f_{t}^{\text {best }}>f_{\text {max }}^{\text {best }} \\ \min _{\text {popsize }}=\text { if } N_{t+1}<\min _{\text {popsize }}\end{cases}$

\section{Results and discussion}

Initially, the equations were generated using regression analysis from observed data as shown in Table 2. The five response parameters have been measured and objective functions are generated as given by Eqs. (12)-(16) for percentage change in surface roughness (R1), hardness (R2), diameter (R3), neck thickness (R4) and stem thickness (R5) respectively.

Surface roughness
From Eqs. (12-16), it is clear that percentage change in surface roughness and hardness should be maximum and while other three parameters i.e. percentage change in diameter, neck thickness and stem thickness should be minimum to achieve optimum solution and these values also should be positive (non-negative). So, to convert the above said problem into pure minimization problem, there is a need to convert the surface roughness and hardness parameters into minimization parameter. Therefore, the fitness functions are redefined as follows:

$$
\begin{aligned}
\mathrm{R} 1= & 242.0+0.2226 \times A-379.5 \times B-0.5195 \times C+5.098 \times D+0.3450 \times E \\
& -6.049 \times F+33.54 \times B \times B+0.000040 \times C \times C-0.1247 \times D \times D-0.000137 \times E \times E \\
& +10.32 \times F \times F-0.1817 \times A \times B+0.3520 \times B \times C+0.007736 \times C \times D \\
& +0.000059 \times C \times E-0.006245 \times D \times E-0.02407 \times E \times F
\end{aligned}
$$

Hardness

$$
\begin{aligned}
\mathrm{R} 2= & 69.48+0.09938 \times A-117.1 \times B-0.1811 \times C+1.699 \times D \\
& +0.09642 \times E+0.2348 \times F+12.62 \times B \times B+0.000015 \times C \times C-0.08035 \times D \times D \\
& -0.000038 \times E \times E+2.335 \times F \times F-0.06317 \times A \times B+0.1068 \times B \times C \\
& +0.003103 \times C \times D+0.000025 \times C \times E-0.001803 \times D \times E-0.007844 \times E \times F
\end{aligned}
$$

Diameter

$$
\begin{aligned}
\mathrm{R} 3= & 23.22+0.03185 \times A-51.58 \times B-0.04622 \times C+0.4197 \times D+0.03238 \times E \\
& -2.126 \times F+15.39 \times B \times B+0.000003 \times C \times C-0.01405 \times D \times D-0.000012 \times E \times E \\
& +1.010 \times F \times F-0.03454 \times A \times B+0.03065 \times B \times C+0.000888 \times C \times D+0.000004 \times C \times E \\
& -0.000690 \times D \times E-0.001110 \times E \times F
\end{aligned}
$$

Neck thickness

$$
\begin{aligned}
\mathrm{R} 4= & -4.322-0.01122 \times A+14.17 \times B+0.01347 \times C-0.1028 \times D-0.01121 \times E \\
& +0.4601 \times F-3.493 \times B \times B-0.000001 \times C \times C+0.003879 \times D \times D+0.000004 \times E \times E \\
& -0.4218 \times F \times F+0.006304 \times A \times B-0.009525 \times B \times C-0.000283 \times C \times D \\
& -0.000001 \times C \times E+0.000176 \times D \times E+0.001022 \times E \times F
\end{aligned}
$$

Stem thickness

$$
\begin{aligned}
\mathrm{R} 5= & -8.374-0.03937 \times A+44.73 \times B+0.03413 \times C-1.071 \times D-0.02883 \times E \\
& +1.017 \times F-15.13 \times B \times B-0.000003 \times C \times C+0.02890 \times D \times D+0.000007 \times E \times E \\
& -1.057 \times F \times F+0.02889 \times A \times B-0.02112 \times B \times C-0.000761 \times C \times D-0.000001 \times C \times E \\
& +0.000746 \times D \times E+0.002250 \times E \times F
\end{aligned}
$$


$f($ Surface roughness $)=$ abs $(1 / \mathrm{R} 1)$

$f$ (Hardness $)=\operatorname{abs}(1 / \mathrm{R} 2)$

$f($ Diameter $)=$ abs $(\mathrm{R} 3)$

$f$ (Neck thickness) $=$ abs (R4)

$f($ Stem thickness $)=$ abs (R5)

The simulations were performed using MATLAB on CORE i7 CPU with 8 GB RAM. The maximum number of iterations and search agents is fixed to 500 and 40 respectively. Moreover, the simulations are repeated for 30 times independently to avoid the random bias and median results are presented as shown in Table 3.

Additionally, the results obtained are compared with the other state-of-the-art algorithms which include ford firefly algorithm (FFA), novel bat algorithm (NBA), GWO, neural network algorithm (NNA), CS, salp swarm algorithm (SSA) and sine cosine algorithm (SCA). The outputs of each algorithm for five response parameters are shown in Table 4. Figure 3 plots convergence curves for fitness values predicted by different optimization algorithms for each response. Since all the responses are must be minimized, it can be noticed that SCAS algorithms is yielding favorable results. For iterations more than 30 , the SCAS is consistently performing best amongst all other conventional algorithms. Hence, the predicted parameters for best fitness values of each response are shown in Table 5. It can be noticed that different parametric settings are recommended by SACS for each response. Furthermore, the finishing cycles predicted by SACS are three each for surface roughness hardness and dimensional accuracy of diameter. In case of accuracy of stem thickness and neck thickness, three finishing cycles are recommended.

SACS attains better results in comparison to the results obtained using FFA, NBA, GWO, CS, SSA and SCA as confirmed by results of Table 4 . However, better results obtained

Table 3 Parameter settings for meta-heuristics

\begin{tabular}{ll}
\hline Algorithm & Parameters \\
\hline FFA & $\mathrm{NP}=40 ; D=6 ; G_{\max }=500 ;$ \\
NBA & $\beta_{0}=1 ; \beta_{\min }=0.2 ; \propto=0.5 ; \gamma=1$ \\
GWO & $\mathrm{NP}=40 ; D=6 ; G_{\max }=500 ; A=0.5 ; r=0.5 ;$ \\
NNA & $\alpha=\gamma=0.9 ; f_{\min }=0 ; f_{\max }=1.5$ \\
CS & $\mathrm{NP}=40 ; D=6 ; G_{\max }=500 ; \mathrm{a}$ \\
& $=[2$ to 0$] ; \mathrm{C}=[0$ to 2$]$ \\
& $\mathrm{NP}=40 ; D=6 ; G_{\max }=500 ; \alpha_{\mathrm{NNA}}=0.99$ \\
& $\mathrm{NP}=40 ; D=6 ; G_{\max }=500 ; p_{a}=0.25$ \\
SSA & $\mathrm{NP}=40 ; D=6 ; G_{\max }=500 ; c_{1}=[2$ to 0$]$ \\
SCA & $\mathrm{NP}=40 ; D=6 ; G_{\max }=500 ; r_{1}=[2$ to0 $]$ \\
SACS & $\mathrm{NP}=40 ; D=6 ; G_{\max }=500 ; p_{m}=$ adaptive \\
\hline
\end{tabular}

NP is number of population, $D$ is dimension of population, $G_{\max }$ is number of iteration by SACS is at the cost of higher computational time taken by SACS as compared to competitive algorithms. The average time taken by SACS is $2.28 \mathrm{~s}$ in comparison to $1.73 \mathrm{~s}, 1.54 \mathrm{~s}$, $2.17 \mathrm{~s}, 1.62 \mathrm{~s}, 1.92 \mathrm{~s}, 2.14 \mathrm{~s}$ for FFA, NBA, GWO, CS, SSA and SCA respectively.

The effect of individual parameter has been studied using main effect plots derived after Taguchi analysis through Minitab Analytical tool. Figure 4a shows the significance of smoothing time (D) and finishing cycles (F) on surface finish of FDM parts. The surface finish is improved with an increase in exposure time and also further improved repeating the cycle. In smoothing chamber, the parts are exposed to heated acetone vapours which melt the upper surface of ABS replicas. Afterwards, these are cooled which resulted in re-settlement of layers due to force of surface tension which resulted in smooth surface $[31,32]$. In case of surface hardness (Fig. 4b), orientation angle $(A)$ and post-cooling time $(E)$ play an important role. The orientation angle of $90^{\circ}$ manifested higher hardness as compared to $90^{\circ}$ which may be attributed to different deposition strategies used during printing at different angles. Also, the cooling at lower temperature for longer time ensures perfect layer settlement which further strengthens the upper surface.

The percentage dimensional accuracy of FDM parts at different locations is significantly affected by two parameters, i.e. orientation angle $(A)$ and smoothing time $(D)$ as shown in Fig. 5. The orientation angle of $90^{\circ}$ yields better dimensional accuracy due to uniform layer deposition as part is suitable oriented to nozzle in this direction. Also, the higher smoothing time improves the accuracy of neck and stem thickness, but it deteriorates the accuracy of diameter. As, the parts are exposed to heated chemical vapours, the layers are resettled and surface is smoothed which results in over all shrinkage of part. Thus, the oversized dimensions tend to acquire original dimensions while already under-sized features are more shrunk due after cooling. In present case, during fabrication, diameter was noted to be under-sized which thickness of two features was over-sized. Thus, the impact of smoothing time is different for these response parameters.

The surface roughness profile and scanning electron microscope images acquired before and after finishing also reveal significant reduction in surface roughness (Fig. 6). The layer resettlement marks are clearly visible in SEM images. The material flows from heightened polymers layers and flows into the voids [33]. After cooling, it gets settled as smooth layers which gives high quality surface. Also, the profile height of layer deposited is significantly reduced after finishing process which confirms the efficacy of this process.

After the prediction of optimized set of parameters, the confirmatory experiments are conducted to validate the 
Table 4 Comparison of output of different algorithms with SACS

\begin{tabular}{|c|c|c|c|c|c|c|}
\hline Parameter & Algorithm & Best & Worst & Average & Median & Standard Deviation \\
\hline \multirow[t]{8}{*}{ Surface roughness } & FFA & $1.03 \times 10^{-02}$ & $1.06 \times 10^{-02}$ & $1.04 \times 10^{-02}$ & $1.04 \times 10^{-02}$ & $3.11 \times 10^{-03}$ \\
\hline & NBA & $1.02 \times 10^{-02}$ & $1.03 \times 10^{-02}$ & $1.02 \times 10^{-02}$ & $1.03 \times 10^{-02}$ & $1.52 \times 10^{-04}$ \\
\hline & GWO & $1.01 \times 10^{-02}$ & $1.01 \times 10^{-02}$ & $1.01 \times 10^{-02}$ & $1.01 \times 10^{-02}$ & $1.63 \times 10^{-05}$ \\
\hline & NNA & $1.01 \times 10^{-02}$ & $1.03 \times 10^{-02}$ & $1.02 \times 10^{-02}$ & $1.02 \times 10^{-02}$ & $1.38 \times 10^{-04}$ \\
\hline & $\mathrm{CS}$ & $1.01 \times 10^{-02}$ & $1.01 \times 10^{-02}$ & $1.02 \times 10^{-02}$ & $1.01 \times 10^{-02}$ & $1.65 \times 10^{-05}$ \\
\hline & SSA & $1.01 \times 10^{-02}$ & $1.01 \times 10^{-02}$ & $1.02 \times 10^{-02}$ & $1.01 \times 10^{-02}$ & $1.32 \times 10^{-05}$ \\
\hline & SCA & $1.01 \times 10^{-02}$ & $1.01 \times 10^{-02}$ & $1.02 \times 10^{-02}$ & $1.01 \times 10^{-02}$ & $1.65 \times 10^{-05}$ \\
\hline & SACS & $1.01 \times 10^{-02}$ & $1.01 \times 10^{-02}$ & $1.02 \times 10^{-02}$ & $1.01 \times 10^{-02}$ & $1.18 \times 10^{-05}$ \\
\hline \multirow[t]{8}{*}{ Hardness } & FFA & $5.72 \times 10^{-02}$ & $5.76 \times 10^{-02}$ & $5.75 \times 10^{-02}$ & $5.74 \times 10^{-02}$ & $6.40 \times 10^{-04}$ \\
\hline & NBA & $5.72 \times 10^{-02}$ & $5.72 \times 10^{-02}$ & $5.72 \times 10^{-02}$ & $5.72 \times 10^{-02}$ & $1.10 \times 10^{-07}$ \\
\hline & GWO & $5.71 \times 10^{-02}$ & $5.72 \times 10^{-02}$ & $5.72 \times 10^{-02}$ & $5.72 \times 10^{-02}$ & $1.38 \times 10^{-07}$ \\
\hline & NNA & $5.72 \times 10^{-02}$ & $5.72 \times 10^{-02}$ & $5.72 \times 10^{-02}$ & $5.72 \times 10^{-02}$ & $1.24 \times 10^{-07}$ \\
\hline & CS & $5.71 \times 10^{-02}$ & $5.72 \times 10^{-02}$ & $5.72 \times 10^{-02}$ & $5.72 \times 10^{-02}$ & $1.10 \times 10^{-07}$ \\
\hline & SSA & $5.71 \times 10^{-02}$ & $5.72 \times 10^{-02}$ & $5.72 \times 10^{-02}$ & $5.72 \times 10^{-02}$ & $1.10 \times 10^{-07}$ \\
\hline & SCA & $5.71 \times 10^{-02}$ & $5.72 \times 10^{-02}$ & $5.72 \times 10^{-02}$ & $5.72 \times 10^{-02}$ & $1.10 \times 10^{-07}$ \\
\hline & SACS & $5.71 \times 10^{-02}$ & $5.72 \times 10^{-02}$ & $5.72 \times 10^{-02}$ & $5.72 \times 10^{-02}$ & $4.49 \times 10^{-08}$ \\
\hline \multirow[t]{8}{*}{ Diameter } & FFA & $1.10 \times 10^{-01}$ & $1.10 \times 10^{-01}$ & $1.10 \times 10^{-01}$ & $1.10 \times 10^{-01}$ & $4.55 \times 10^{-05}$ \\
\hline & NBA & $1.10 \times 10^{-01}$ & $1.10 \times 10^{-01}$ & $1.10 \times 10^{-01}$ & $1.10 \times 10^{-01}$ & $1.52 \times 10^{-04}$ \\
\hline & GWO & $1.10 \times 10^{-01}$ & $1.10 \times 10^{-01}$ & $1.10 \times 10^{-01}$ & $1.10 \times 10^{-01}$ & $7.49 \times 10^{-05}$ \\
\hline & NNA & $1.10 \times 10^{-01}$ & $1.10 \times 10^{-01}$ & $1.10 \times 10^{-01}$ & $1.10 \times 10^{-01}$ & $5.24 \times 10^{-05}$ \\
\hline & $\mathrm{CS}$ & $1.10 \times 10^{-01}$ & $1.10 \times 10^{-01}$ & $1.10 \times 10^{-01}$ & $1.10 \times 10^{-01}$ & $3.29 \times 10^{-06}$ \\
\hline & SSA & $1.10 \times 10^{-01}$ & $1.10 \times 10^{-01}$ & $1.10 \times 10^{-01}$ & $1.10 \times 10^{-01}$ & $4.55 \times 10^{-06}$ \\
\hline & SCA & $1.10 \times 10^{-01}$ & $1.10 \times 10^{-01}$ & $1.10 \times 10^{-01}$ & $1.10 \times 10^{-01}$ & $1.21 \times 10^{-05}$ \\
\hline & SACS & $1.10 \times 10^{-01}$ & $1.10 \times 10^{-01}$ & $1.10 \times 10^{-01}$ & $1.10 \times 10^{-01}$ & $8.86 \times 10^{-07}$ \\
\hline \multirow[t]{8}{*}{ Neck thickness } & FFA & $3.00 \times 10^{-02}$ & $3.00 \times 10^{-02}$ & $3.00 \times 10^{-02}$ & $3.00 \times 10^{-02}$ & $1.96 \times 10^{-07}$ \\
\hline & NBA & $3.00 \times 10^{-02}$ & $3.00 \times 10^{-02}$ & $3.00 \times 10^{-02}$ & $3.00 \times 10^{-02}$ & $6.39 \times 10^{-05}$ \\
\hline & GWO & $3.00 \times 10^{-02}$ & $3.00 \times 10^{-02}$ & $3.00 \times 10^{-02}$ & $3.00 \times 10^{-02}$ & $1.35 \times 10^{-05}$ \\
\hline & NNA & $2.99 \times 10^{-02}$ & $3.00 \times 10^{-02}$ & $3.00 \times 10^{-02}$ & $3.00 \times 10^{-02}$ & $1.35 \times 10^{-05}$ \\
\hline & $\mathrm{CS}$ & $3.00 \times 10^{-02}$ & $3.00 \times 10^{-02}$ & $3.00 \times 10^{-02}$ & $3.00 \times 10^{-02}$ & $1.87 \times 10^{-06}$ \\
\hline & SSA & $3.00 \times 10^{-02}$ & $3.00 \times 10^{-02}$ & $3.00 \times 10^{-02}$ & $3.00 \times 10^{-02}$ & $4.35 \times 10^{-06}$ \\
\hline & SCA & $3.00 \times 10^{-02}$ & $3.01 \times 10^{-02}$ & $3.00 \times 10^{-02}$ & $3.00 \times 10^{-02}$ & $2.23 \times 10^{-04}$ \\
\hline & SACS & $2.99 \times 10^{-02}$ & $3.00 \times 10^{-02}$ & $3.00 \times 10^{-02}$ & $3.00 \times 10^{-02}$ & $6.69 \times 10^{-09}$ \\
\hline \multirow[t]{8}{*}{ Stem thickness } & FFA & $4.00 \times 10^{-02}$ & $4.01 \times 10^{-02}$ & $4.00 \times 10^{-02}$ & $4.00 \times 10^{-02}$ & $1.01 \times 10^{-06}$ \\
\hline & NBA & $4.00 \times 10^{-02}$ & $4.02 \times 10^{-02}$ & $4.00 \times 10^{-02}$ & $4.00 \times 10^{-02}$ & $5.66 \times 10^{-05}$ \\
\hline & GWO & $4.00 \times 10^{-02}$ & $4.02 \times 10^{-02}$ & $4.00 \times 10^{-02}$ & $4.00 \times 10^{-02}$ & $3.81 \times 10^{-05}$ \\
\hline & NNA & $4.00 \times 10^{-02}$ & $4.01 \times 10^{-02}$ & $4.00 \times 10^{-02}$ & $4.00 \times 10^{-02}$ & $3.15 \times 10^{-05}$ \\
\hline & $\mathrm{CS}$ & $4.00 \times 10^{-02}$ & $4.01 \times 10^{-02}$ & $4.00 \times 10^{-02}$ & $4.00 \times 10^{-02}$ & $3.18 \times 10^{-06}$ \\
\hline & SSA & $4.00 \times 10^{-02}$ & $4.01 \times 10^{-02}$ & $4.00 \times 10^{-02}$ & $4.00 \times 10^{-02}$ & $1.38 \times 10^{-05}$ \\
\hline & SCA & $4.00 \times 10^{-02}$ & $4.03 \times 10^{-02}$ & $4.00 \times 10^{-02}$ & $4.00 \times 10^{-02}$ & $1.09 \times 10^{-03}$ \\
\hline & SACS & $4.00 \times 10^{-02}$ & $4.01 \times 10^{-02}$ & $4.00 \times 10^{-02}$ & $4.00 \times 10^{-02}$ & $1.85 \times 10^{-08}$ \\
\hline
\end{tabular}

results generated by SACS algorithm. Conclusively, five parts were fabricated and finished at optimized settings for each response as per Table 5. The output in terms of percentage change in surface roughness, hardness and dimensional accuracy was measured and compared with predicted results as shown in Table 6. It was observed that optimized set of parameters yielded best results for all the response parameters and experimental results were very close to predicted results.

\subsection{Corollary}

The corollary has been presented to check the accuracy of results generated by SACS algorithm. Equation (12) for percentage change in surface roughness is considered for investigation. 

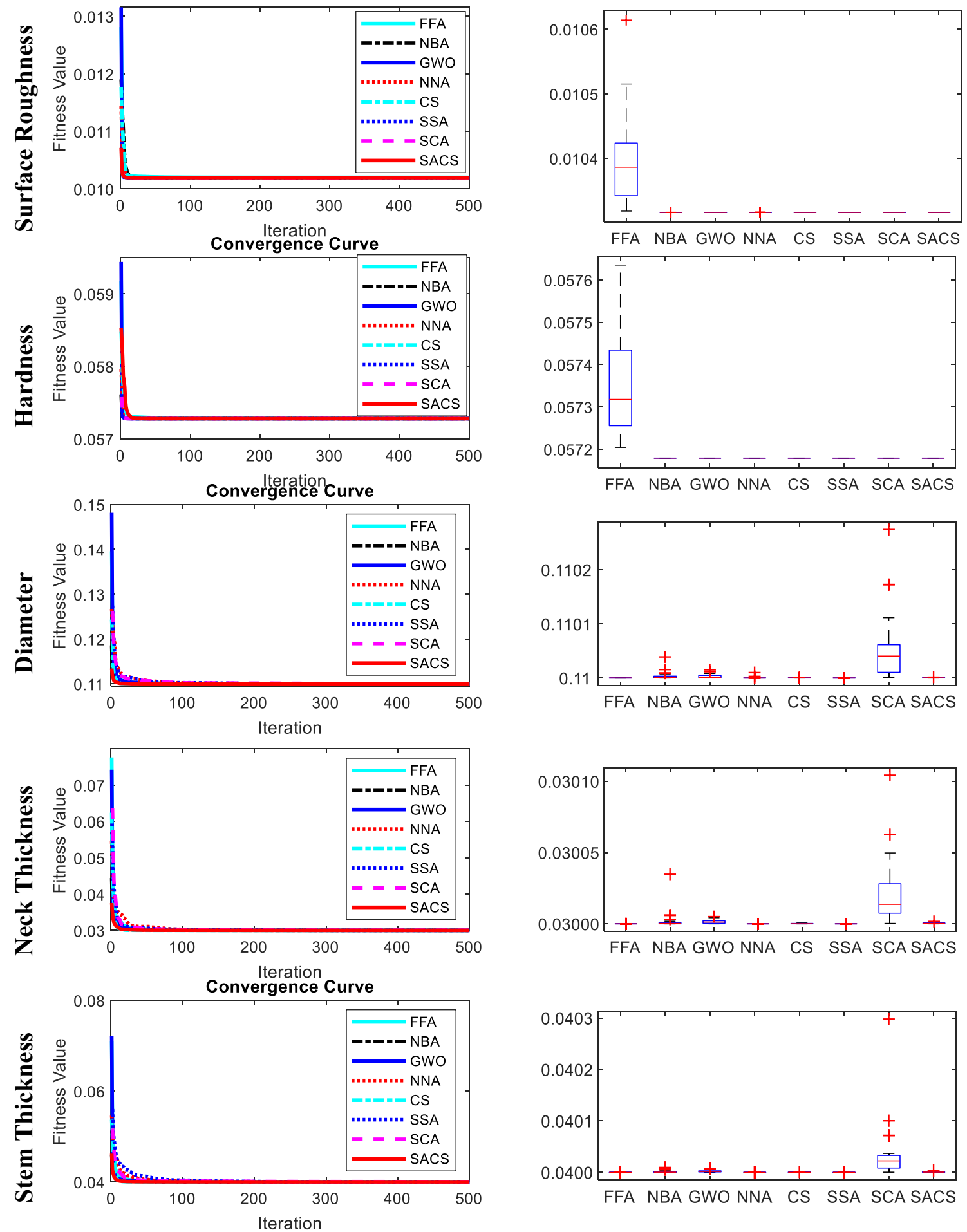

Fig. 3 Comparison of convergence curves and box plots for different algorithms 
Table 5 Optimized parameter settings for each response suggested by SACS
Fig. 4 Main effect plots for percentage change in a surface roughness, b hardness

\begin{tabular}{|c|c|c|c|c|c|c|}
\hline Parameter & $\begin{array}{l}A \\
\left({ }^{\circ}\right)\end{array}$ & $\begin{array}{l}B \\
\left(\mathrm{~g} / \mathrm{cm}^{3}\right)\end{array}$ & $\begin{array}{l}C \\
(\mathrm{~s})\end{array}$ & $\begin{array}{l}D \\
(\mathrm{~s})\end{array}$ & $\begin{array}{l}E \\
(\mathrm{~s})\end{array}$ & $F$ \\
\hline Surface roughness & 0 & 0.9457 & 600 & 30 & 1635 & 3 \\
\hline Hardness & 0 & 0.9457 & 1200 & 30 & 1700 & 3 \\
\hline Diameter & 60 & 0.73033 & 1650 & 35.2143 & 1643 & 2 \\
\hline Neck thickness & 45 & 0.9429 & 847.3688 & 33.0331 & 707.4584 & 2 \\
\hline Stem thickness & 45.6 & 0.6395 & 1100.2993 & 17.5186 & 987.2629 & 2 \\
\hline
\end{tabular}

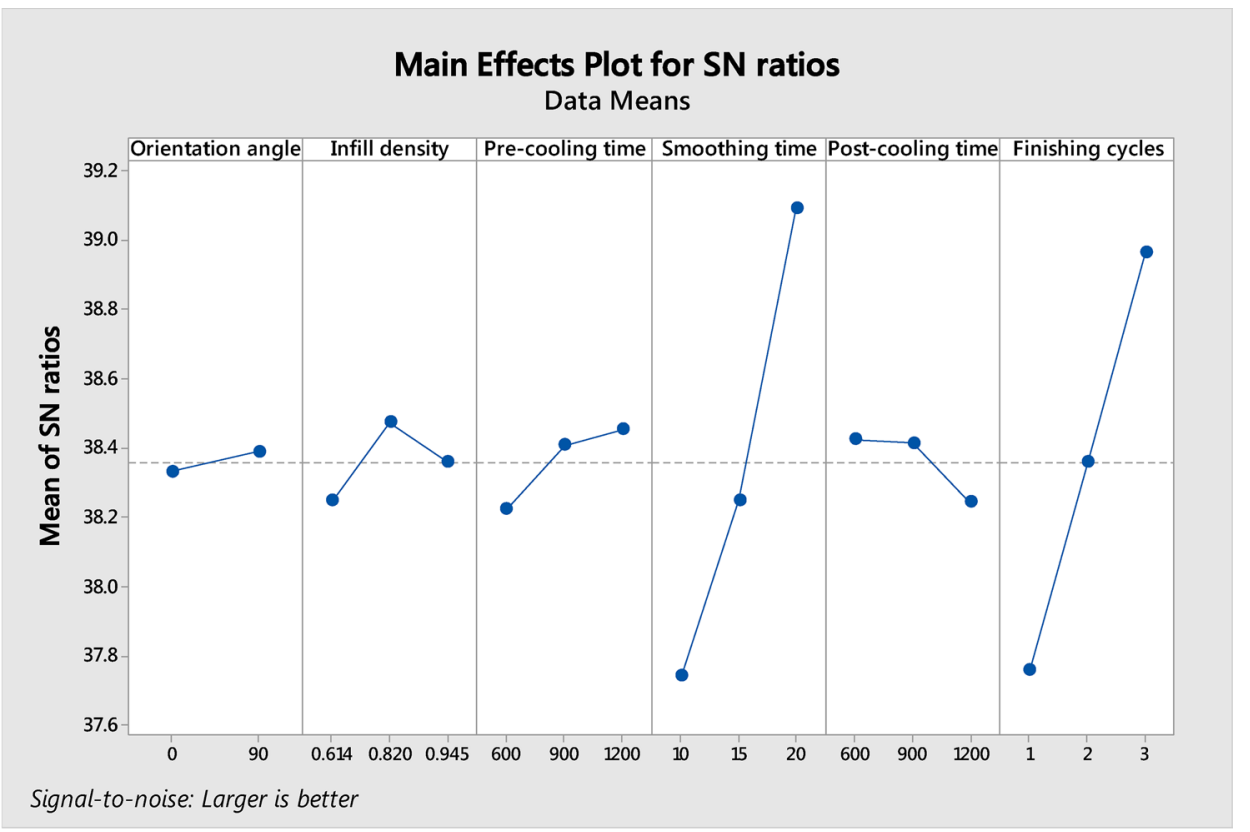

(a)

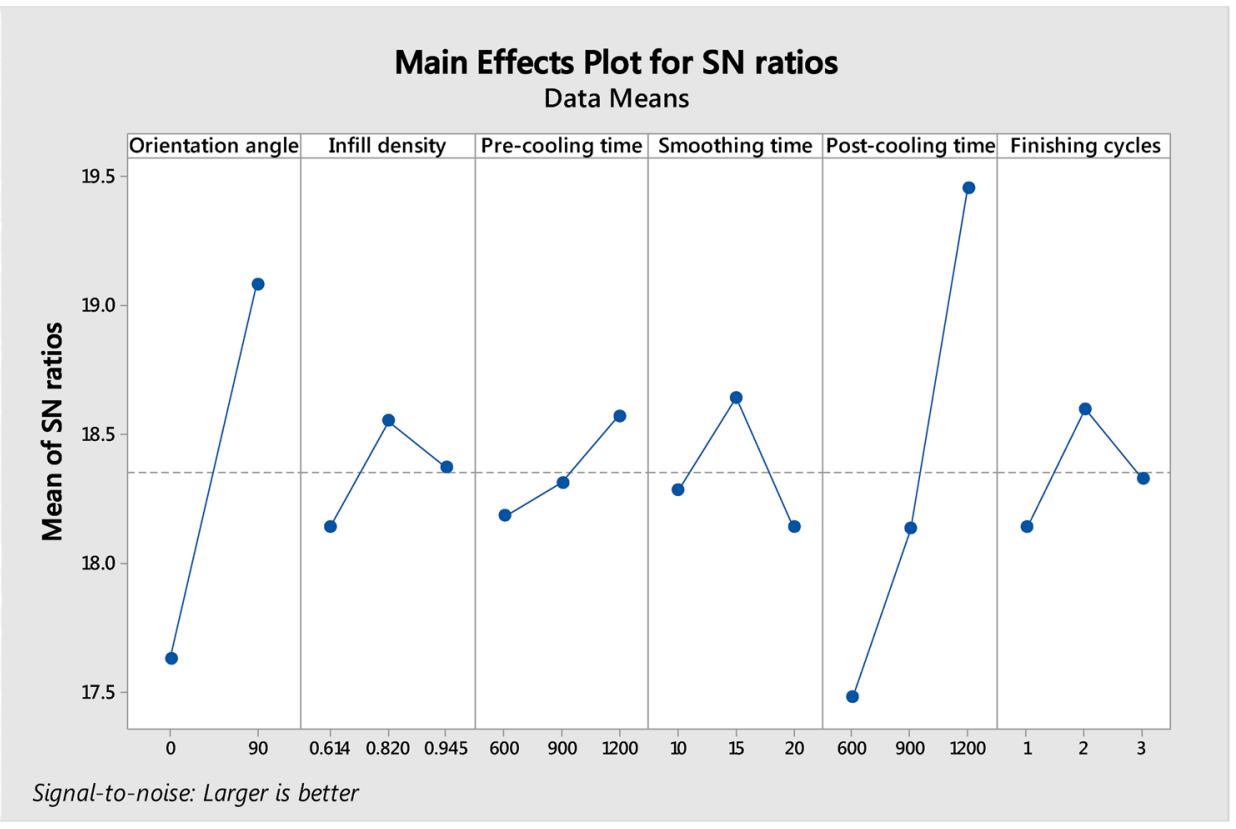

(b) 
Fig. 5 Main effect plots for percentage change in a diameter, $\mathbf{b}$ neck thickness, $\mathbf{c}$ stem thickness

\section{Main Effects Plot for SN ratios} Data Means

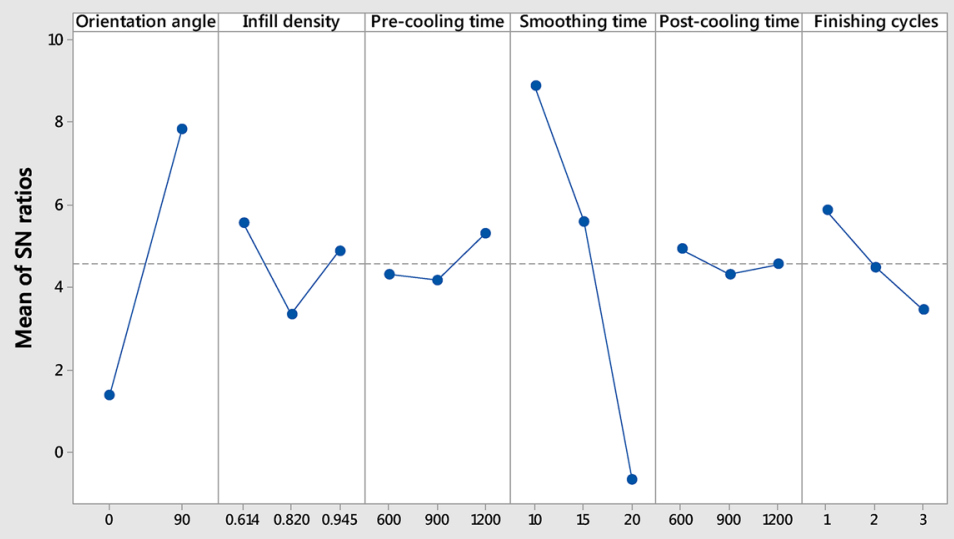

Signal-to-noise: Smaller is better

(a)

\section{Main Effects Plot for SN ratios} Data Means

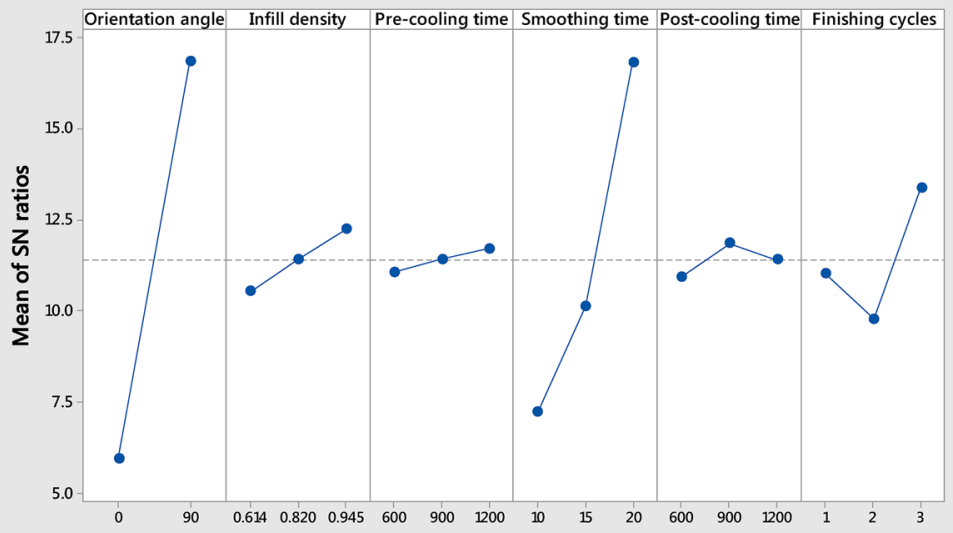

Signal-to-noise: Smaller is better

(b)

Main Effects Plot for SN ratios Data Means

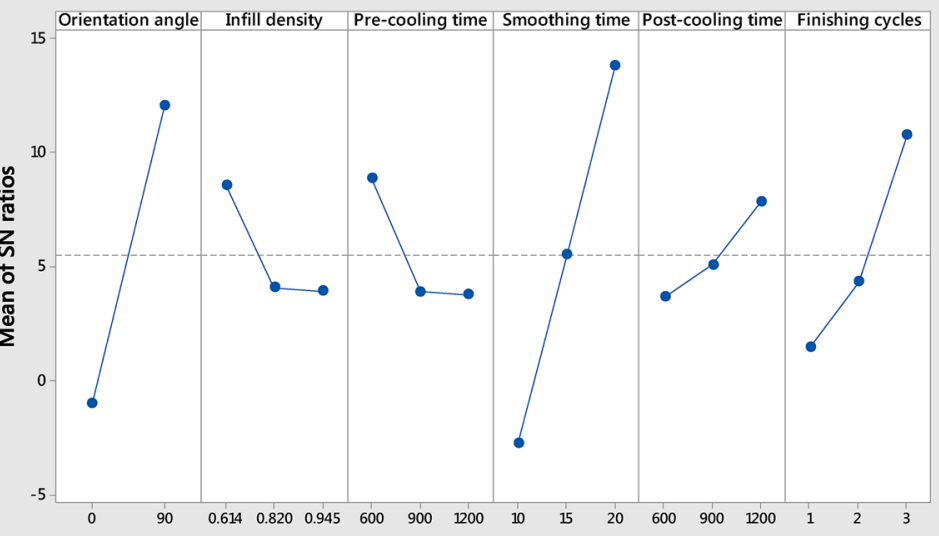

Signal-to-noise: Smaller is better

(c) 

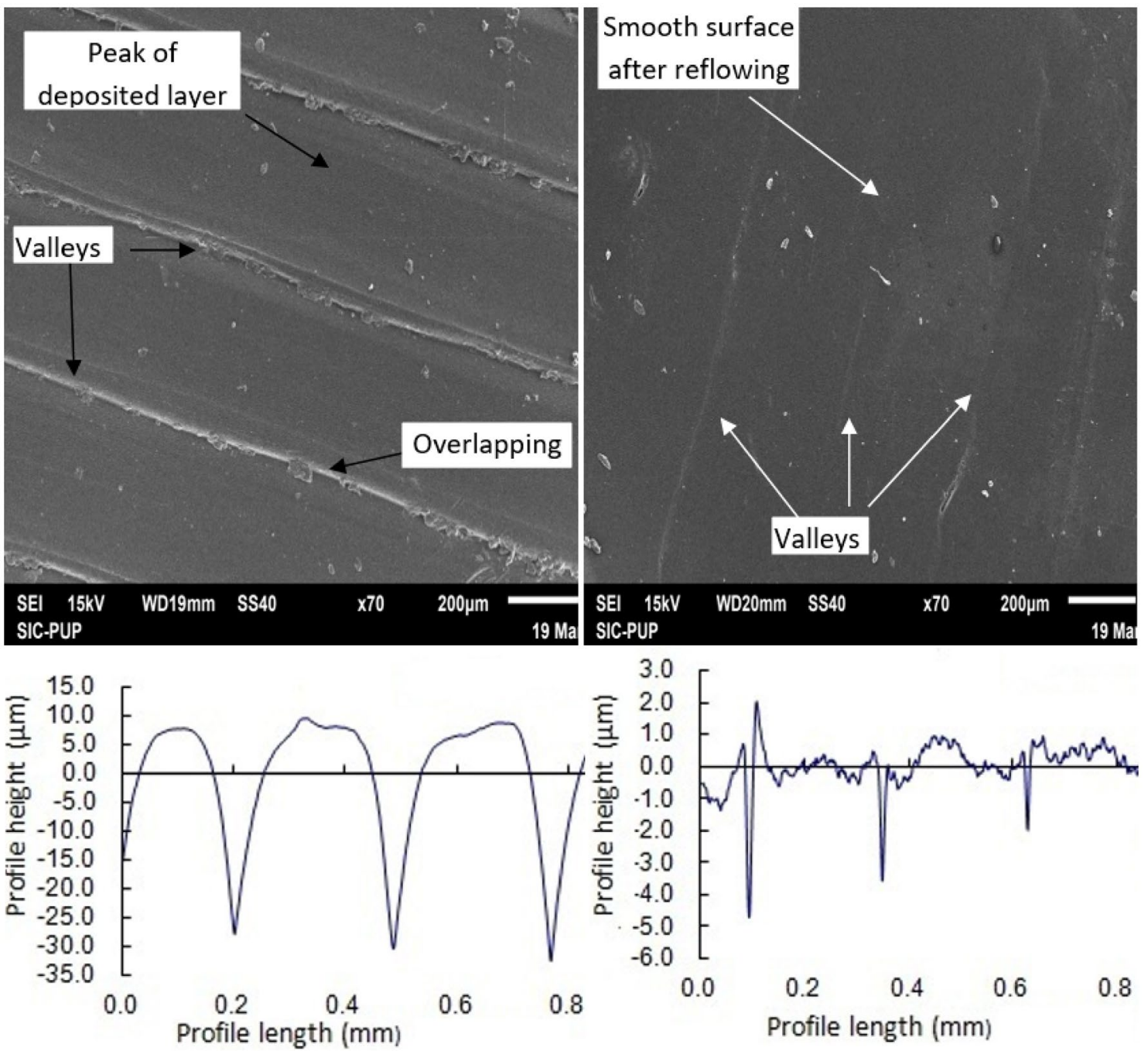

Fig. 6 Scanning electron microscope and surface roughness profiles before and after finishing

Table 6 Comparison of predicted and experimental results for each response

\begin{tabular}{llc}
\hline Response & Experimental & Predicted \\
\hline Percentage change in Surface Roughness & 98.12 & 98.43 \\
Percentage change in Hardness & 17.85 & 19.27 \\
Percentage change in Diameter & 0.16 & 0.1824 \\
Percentage change in Neck Thickness & 0.04 & 0.0389 \\
Percentage change in Stem Thickness & 0.05 & 0.0142 \\
\hline
\end{tabular}

$$
\begin{aligned}
\mathrm{R} 1= & 242.0+0.2226 \times A-379.5 \times B-0.5195 \\
& \times C+5.098 \times D+0.3450 \times E \\
& -6.049 \times F+33.54 \times B \times B+0.000040 \\
& \times C \times C-0.1247 \times D \times D \\
& -0.000137 \times E \times E+10.32 \\
& \times F \times F-0.1817 \times A \times B+0.3520 \\
& \times B \times C+0.007736 \times C \times D+0.000059 \times C \times E \\
& -0.006245 \times D \times E-0.02407 \times E \times F
\end{aligned}
$$


The predicted values of parameters $A, B, C, D, E, F$ are inserted in above equation from Table 5 for surface roughness.

$$
\begin{aligned}
\mathrm{R} 1= & 242.0+0.2226 \times 0-379.5 \times 0.9457 \\
& -0.5195 \times 600+5.098 \times 30+0.3450 \times 1635 \\
& -6.049 \times 3+33.54 \times 0.9457 \times 0.9457 \\
& +0.000040 \times 600 \times 600-0.1247 \times 30 \times 30 \\
& -0.000137 \times 1635 \times 1635+10.32 \times 3 \times 3 \\
& -0.1817 \times 0 \times 0.9457+0.3520 \times 0.9457 \times 600 \\
& +0.007736 \times 600 \times 30+0.000059 \times 600 \times 1635 \\
& -0.006245 \times 30 \times 1635-0.02407 \times 1635 \times 3
\end{aligned}
$$

After calculations, it can be written as:

$$
\begin{aligned}
\mathrm{R} 1= & 242+0-358.89-311.7 \\
& +152.94+564.075-18.147+29.9964+14.4 \\
& -112.23-366.2318+92.88-0 \\
& +199.73+139.248+57.879 \\
& -306.3172-118.0633 \\
\mathrm{R} 1 & =98.43 \text { (predicted) }
\end{aligned}
$$

Hence, the percentage change in surface roughness $(98.43 \%)$ predicted by SACS algorithm is very near to the experimental value (98.12\%). Similarly, other response parameters can be calculated and compared with experimental results.

\section{Conclusion}

The efficiency of advanced algorithm for finding solutions to optimization problems in additive manufacturing has been demonstrated in this study. The chemical finishing process has been successfully used for surface quality enhancement of FDM parts. But selection of optimum set of parameters is major issue which must be resolved. This study outlines the combined parameters of FDM and chemical finishing processes using SACS algorithm to achieve best surface finish, hardness and dimensional accuracy. The results of proposed algorithm have been compared with other optimization algorithms, and it was found that best results were achieved in terms of surface finish, hardness and dimensional accuracy of FDM parts.

\section{Declarations}

Conflict of interest The authors declare no conflict of interest.

\section{References}

1. Boschetto A, Bottini L (2015) Roughness prediction in coupled operations of fused deposition modeling and barrel finishing. $\mathbf{J}$ Mater Process Technol 219:181-192. https://doi.org/10.1016/j. jmatprotec.2014.12.021

2. Peng A, Xiao X, Yue R (2014) Process parameter optimization for fused deposition modeling using response surface methodology combined with fuzzy inference system. Int J Adv Manuf Tech 73:87-100. https://doi.org/10.1007/s00170-014-5796-5

3. Vasudevarao B, Natarajan DP, Henderson M (2000) Sensitivity of RP surface finish to process parameter variation. In: Proc. of 11th solid freeform fabrication symposium, Austin, pp 252-58

4. Dudek P (2013) FDM 3D printing technology in manufacturing composite elements. Arch Metall Mater 58(4):1415-1418. https:// doi.org/10.2478/amm-2013-0186

5. Melocchi A, Briatico-Vangosa F, Uboldi M, Parietti F, Turchi M, von Zeppelin D, Zidan A et al (2021) Quality considerations on the pharmaceutical applications of fused deposition modeling 3D printing. Int J Pharm 592:119901

6. Tareq MS, Rahman T, Hossain M, Dorrington P (2021) Additive manufacturing and the COVID-19 challenges: An in-depth study. J Manuf Syst 2021:1

7. Longhitano GA, Nunes GB, Candido G, da Silva JVL (2021) The role of 3D printing during COVID-19 pandemic: a review. Progress Addit Manuf 6(1):19-37

8. Calì M, Pascoletti G, Gaeta M, Milazzo G, Ambu R (2020) New filaments with natural fillers for FDM 3D printing and their applications in biomedical field. Procedia Manuf 51:698-703

9. Sathies T, Senthil P, Anoop MS (2020) A review on advancements in applications of fused deposition modelling process. Rapid Prototyp J 2020:5

10. Jain P, Kuthe AM (2013) Feasibility study of manufacturing using rapid prototyping: FDM approach. Procedia Eng 63:4-11

11. Singh TB, Chohan JS, Kumar R (2020) Performance analysis of vapour finishing apparatus for surface enhancement of FDM parts. Mater Today Proc 26(3):3497-3502

12. Kumar A, Alam Z, Khan DA, Jha S (2019) Nanofinishing of FDM-fabricated components using ball end magnetorheological finishing process. Mater Manuf Process 34(2):232-242

13. Mali HS, Prajwal B, Gupta D, Kishan J (2018) Abrasive flow finishing of FDM printed parts using a sustainable media. Rapid Prototyp J 2018:5

14. Jayanth N, Senthil P, Prakash C (2018) Effect of chemical treatment on tensile strength and surface roughness of 3D-printed ABS using the FDM process. Virt Phys Prototyp 13(3):155-163

15. Gajdoš I, Spišák E, Kaščák L, Krasinskyi V (2015) Surface finish techniques for FDM parts. In: Materials science forum (vol 818, pp 45-48). Trans Tech Publications Ltd

16. Perez Dewey M, Ulutan D (2017) Development of laser polishing as an auxiliary post-process to improve surface quality in fused deposition modeling parts. In: International manufacturing science and engineering conference ( $v o l$ 50732, p V002T01A006). American Society of Mechanical Engineers

17. Roach DJ, Roberts C, Wong J, Kuang X, Kovitz J, Zhang Q, Qi $\mathrm{HJ}$ et al (2020) Surface modification of fused filament fabrication (FFF) 3D printed substrates by inkjet printing polyimide for printed electronics. Addit Manuf 36:101544

18. Saad MS, Nor AM, Baharudin ME, Zakaria MZ, Aiman AF (2019) Optimization of surface roughness in FDM 3D printer using response surface methodology, particle swarm optimization, and symbiotic organism search algorithms. Int J Adv Manuf Technol 105(12):5121-5137 
19. Boschetto A, Giordano V, Veniali F (2013) Surface roughness prediction in fused deposition modelling by neural networks. Int J Adv Manuf Technol 67(9-12):2727-2742

20. Mahapatra SS, Sood AK (2012) Bayesian regularization-based Levenberg-Marquardt neural model combined with BFOA for improving surface finish of FDM processed part. Int J Adv Manuf Technol 60(9-12):1223-1235

21. Vahabli E, Rahmati S (2017) Improvement of FDM parts' surface quality using optimized neural networks-medical case studies. Rapid Prototyp J 23:4

22. Raju M, Gupta MK, Bhanot N, Sharma VS (2019) A hybrid PSOBFO evolutionary algorithm for optimization of fused deposition modelling process parameters. J Intell Manuf 30(7):2743-2758

23. ISO Standard (1987) ISO 4287/1 Surface roughness-terminologypart 1: surface and its Parameters

24. American Society for Testing and Materials (2010) ASTM D224005 standard test method for rubber property: durometer hardness. In: West Conshohocken: ASTM, 2010

25. ISO 10360-2: 2009 (2009) Geometrical product specifications (GPS)-acceptance and reverification tests for coordinate measuring machines (CMM)-part 2: CMMs used for measuring linear dimensions

26. Kennedy J (2003) Bare bones particle swarms. In: Swarm Intelligence Symposium, 2003. SIS'03. Proceedings of the 2003 IEEE, pp 80-87
27. Scholz F (2008) Inference for the Weibull distribution, Stat 498B Industrial Statistics 632, pp 6-10

28. Salgotra R, Singh U, Saha S (2018) New cuckoo search algorithms with enhanced exploration and exploitation properties. Expert Syst Appl 95:384-420

29. Salgotra R, Singh U, Sharma S (2019) On the improvement in grey wolf optimization. Neural Comput Appl 2019:1-40

30. Salgotra R, Singh U, Saha S, Gandomi AH (2020) Self-adaptive cuckoo search algorithm: analysis and experimentation. Swarm Evol Comput 60:100751. https://doi.org/10.1016/j.swevo.2020. 100751

31. Taufik M, Jain PK (2020) Thermally assisted finishing of fused deposition modelling build part using a novel CNC tool. J Manuf Process 59:266-278

32. Garg A, Bhattacharya A, Batish A (2017) Effect of cold vapour treatment on geometric accuracy of fused deposition modelling parts. Rapid Prototyp J 2017:54

33. Mu M, Ou CY, Wang J, Liu Y (2020) Surface modification of prototypes in fused filament fabrication using chemical vapour smoothing. Addit Manuf 31:100972

Publisher's Note Springer Nature remains neutral with regard to jurisdictional claims in published maps and institutional affiliations. 